\title{
Validation of Physics Models of Geant4 using data from CMS Experiment
}

\author{
CMS Collaboration
}




\section{Introduction}

- CMS Simulation application is based on Geant4 and CMS has moved with newer versions to benefit from better physics and software performance: 2015 Geant4 10.0p02

- CMS used the version Geant4.10.4.p03 since 2018 for its legacy MC production for Run2 data sets

2017 Geant4 10.2p02

2018 Geant4 10.4p03

- CMS used the version Geant4.10.6.p02 since 2020

2021 Geant4 10.7p01

- CMS has now moved to the version Geant4.10.7.p01 for Run3 MC production. I nis version is available since February, 2021.

- Adaptation of a new Geant4 version or a new Physics List requires validation of the model predictions with some of the existing data

- The validation is carried out using 2 sources of data:

- 2006 test beam with CMS calorimeter prototypes (hadron beams of different types and different energies)

- Collision data from the CMS experiment utilizing zero bias or minimum bias triggers from low luminosity runs

- Similar comparisons were done earlier and the comparisons were used to improve the quality of Geant4 predictions 


\section{Geant4 in CMS}

- CMS is planning to use the same physics list for ultra legacy as well as Run3 production

- FTFP_BERT_EMM

- The list FTFP_BERT uses FTFP and Bertini Cascade models with slightly different transition regions in the two versions. For the version Geant4.10.4.p03:

- Bertini Cascade valid for energies $\leq 12 \mathrm{GeV}$

- FTFP valid at $\geq 3 \mathrm{GeV}$

and in version Geant4.10.6.p02 and Geant4.10.7.p01:

- Bertini Cascade valid at $\leq 12 \mathrm{GeV}$ for pions and $\leq 6 \mathrm{GeV}$ for all other hadrons

- FTFP valid at $\geq 3 \mathrm{GeV}$

- EMM specify the physics models for electromagnetic processes

- EMM uses the default multiple scattering model for regions of the sampling calorimeters (HCAL and HGCAL) and a simplified multiple scattering model elsewhere

- Coefficients of Birk's law for plastic scintillator are retuned for the versions Geant4.10.6.p02 and Geant4.10.7.p01

- Default values for Birk's constants for HCAL used to be - $\mathrm{C} 1=0.0052 ; \mathrm{C} 2=0.142$

- The tuned set is $\bullet \mathrm{C} 1=0.006 ; \mathrm{C} 2=0.142$

$$
\left[1+C_{1} \cdot\left(\frac{d E}{d x}\right)+C_{2} \cdot\left(\frac{d E}{d x}\right)^{2}\right]^{-1}
$$



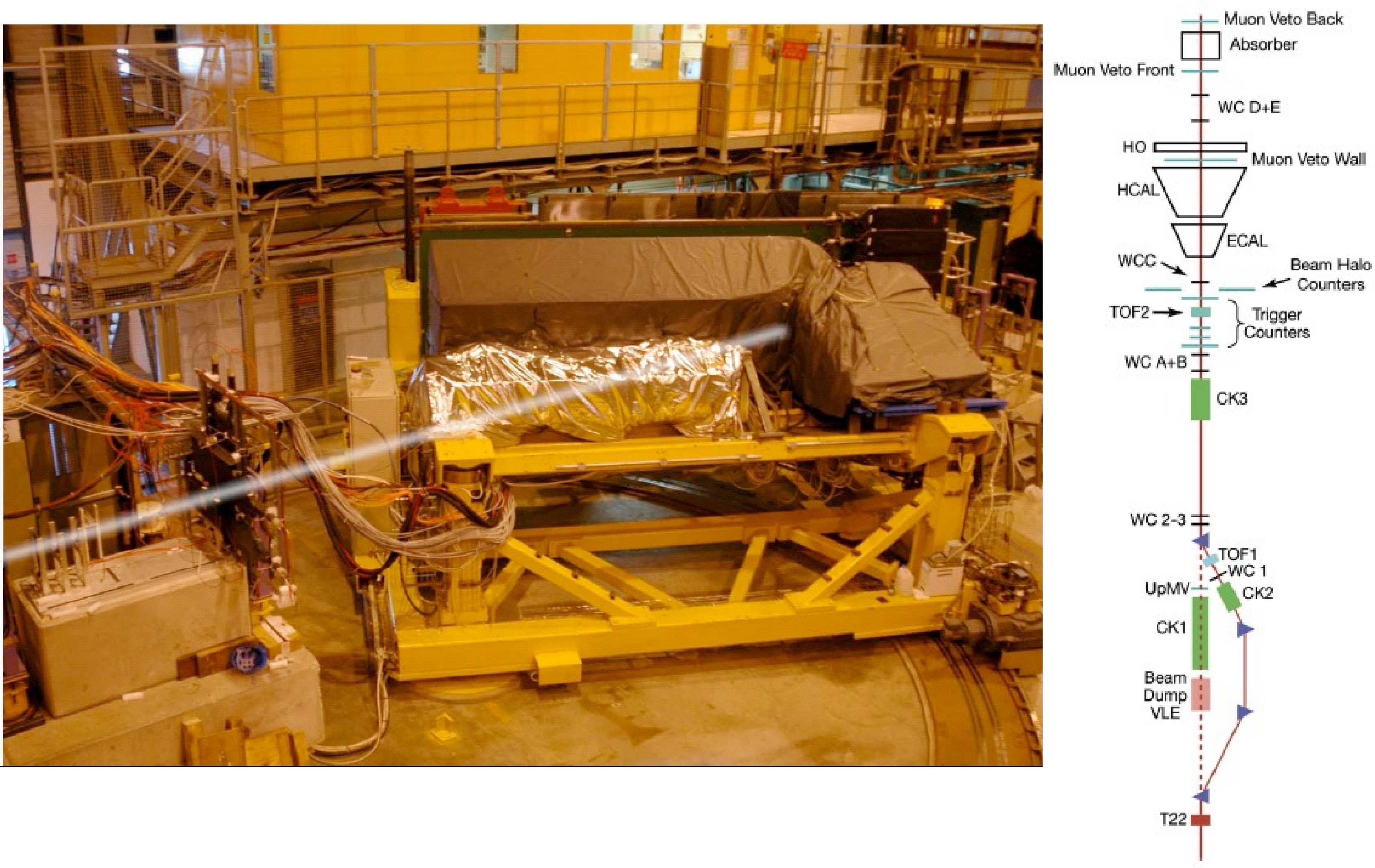

S. Banerjee, V. Ivantchenko 


\section{TestBeam Data}

- CMS collected data with prototype modules of Hadron Calorimeter and a supermodule of the barrel Electromagnetic Calorimeter in the $\mathrm{H} 2$ test beam area at CERN during 2006.

- Special action was taken to go to low energy hadron beam down to $1 \mathrm{GeV}$ using a secondary target

- The analysis utilized particle identification using data from TOF counters and Cherenkov detectors up to energy of $9 \mathrm{GeV}$

- The results consist of mean energy response (measured as the ratio of the total energy in the calorimeter to the beam momentum) as a function of beam momentum for different beam types, the energy resolution and some energy distributions for particles of a given type at a given momentum

- Results from this test beam were published and used in many comparisons presented in earlier conference 


\section{Mean response for $\pi$ and proton}
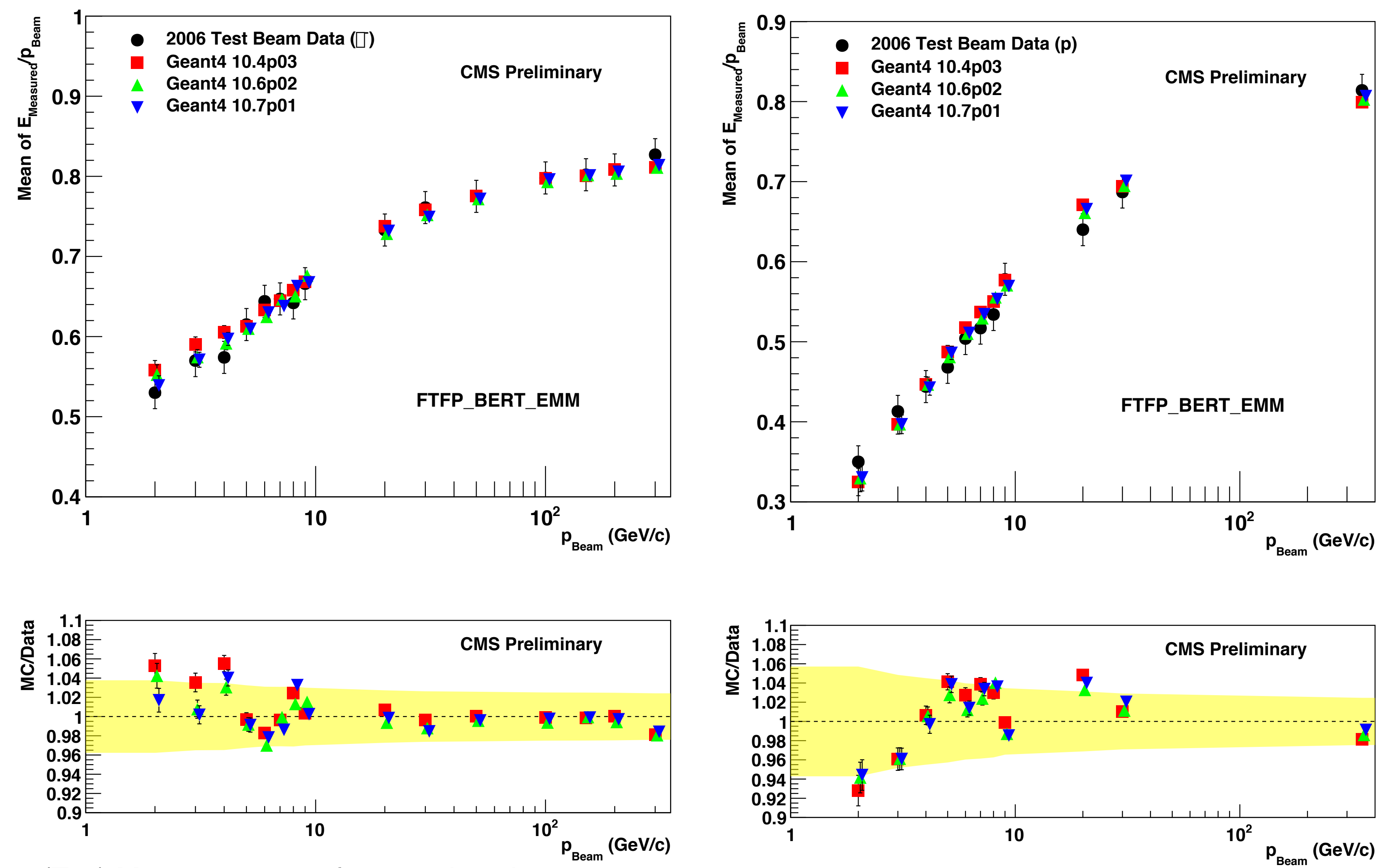

(Top) Mean response for negative pions as a function of momentum compared to $\mathrm{MC}$ predictions; (bottom) Ratio of MC to data for vCHEP 209 ative pions as a function of momentum

(Top) Mean response for protons as a function of momentum compared to MC predictions; (bottom) Ratio of MC to data for protons as a 6 function of momentum 


\section{Summary from Mean Response}

\section{$\mathrm{X}^{2} / \mathrm{d}$.o.f. between data and Monte Carlo}

$\begin{array}{ccccccc} & \begin{array}{c}\text { negative } \\ \text { pions }\end{array} & \begin{array}{c}\text { positive } \\ \text { pions }\end{array} & \begin{array}{c}\text { negative } \\ \text { kaons }\end{array} & \begin{array}{c}\text { positive } \\ \text { kaons }\end{array} & \begin{array}{c}\text { protons } \\ \text { protons }\end{array} \\ \begin{array}{c}\text { G4 10.4.p03 } \\ \text { FTFP_BERT_EMM }\end{array} & 0.45 & 0.73 & 26.2 & 26.8 & 0.80 & 1.78 \\ \begin{array}{c}\text { G4 10.6.p02 } \\ \text { FTFP_BERT_EMM }\end{array} & 0.31 & 1.14 & 19.4 & 14.4 & 0.53 & 1.81 \\ \begin{array}{c}\text { G10.7.p01 } \\ \text { FTFP_BERT_EMM }\end{array} & 0.28 & 0.93 & 20.8 & 14.9 & 0.63 & 3.84\end{array}$

- Level of agreement is good for pions and protons, while it is not good for kaons. Response for pions and kaons are very similar in the data but not in MC.

- The predictions from 10.6.p02 and 10.7.p01 show some improvement for kaons, some deterioration for anti-protons, and acceptable agreement for pions, and protons

- pp collisions at high energies produce mostly pions. So one expects to have a reasonable agreement between data and $\mathrm{MC}$ with the current physics list in the Geant4 versions 10.6.p02 and 10.7.p01 


\section{Energy Resolutions for $m$ and proton 1}
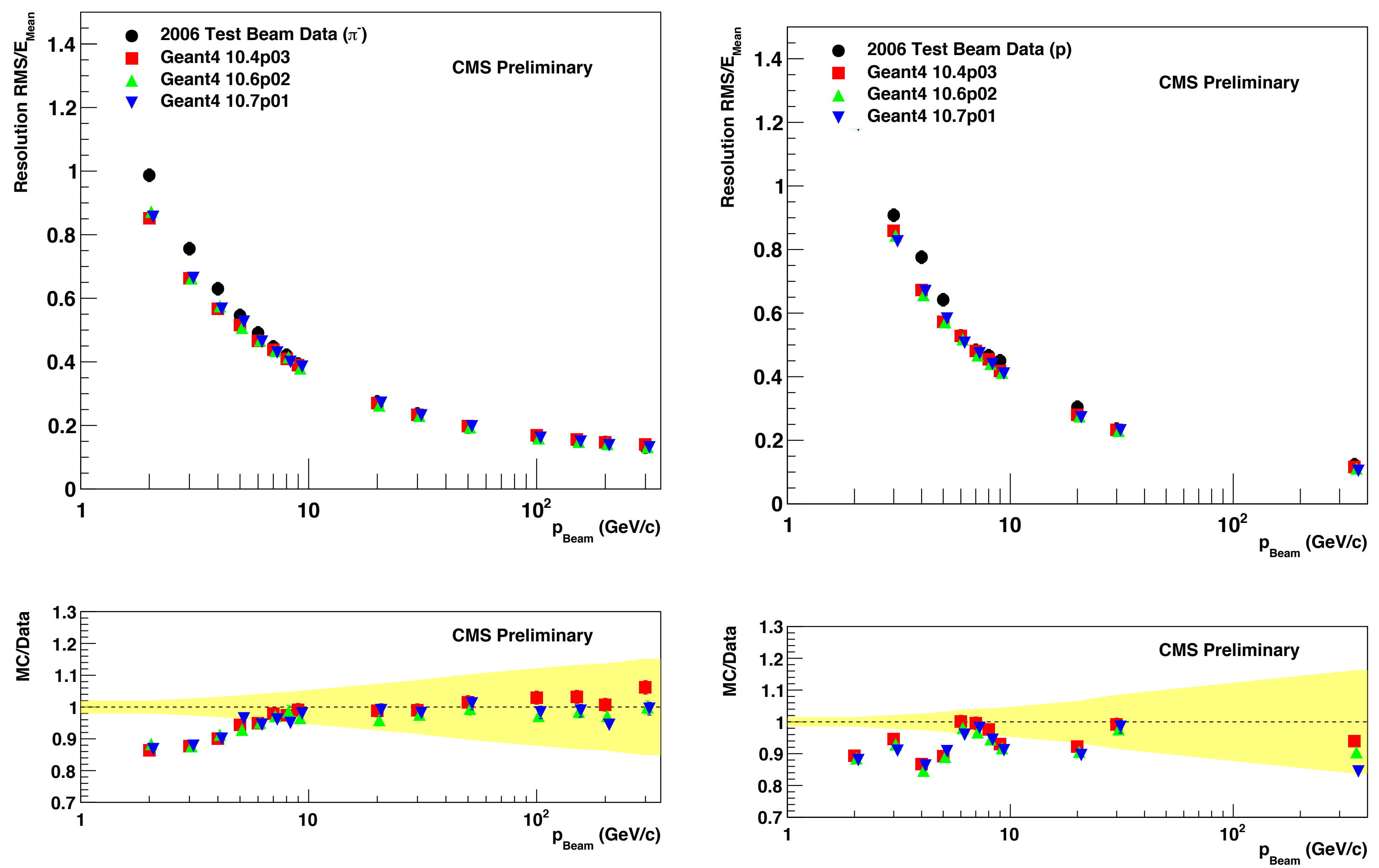

Energy resolution for negative pions as a function of momentum (top) and ratio of MC to data (bottom)

Energy resolution for protons as a function of momentum (top) and ratio of $\mathrm{MC}$ to data (bottom) 


\section{Isolated Charged Particles}

- Compare ratio of calorimeter energy measurement to track momentum for isolated charged hadrons between data and MC

- Select good charged tracks reaching the calorimeter surface

- Impose isolation of these charged particles

- propagate track to calorimeter surface and study momentum of tracks (selected with looser criteria) reaching ECAL (HCAL) within a matrix of $31 \times 31$ (7x7) around the impact point of the selected track. Demand no other track in the isolation region.

- study energy deposited in an annular region in ECAL (HCAL) between 15x15 and $11 \times 11$ (7x7 and 5x5) matrices for neutral isolation. Demand energy in either annular region to be less than $2 \mathrm{GeV}$

- Measure the energy in a matrix of NxN cells around the point of impact. Two versions of NxN matrix are defined for ECAL and HCAL

- ECAL uses $7 \times 7$ or $11 \times 11$ matrix

- HCAL uses $3 \times 3$ or $5 \times 5$ matrix

- The methodology was developed using $7 \mathrm{TeV}$ data and analysis of the 2016 low pileup data plus the comparisons with earlier Geant4 model predictions were presented in a few earlier CHEP conferences. 


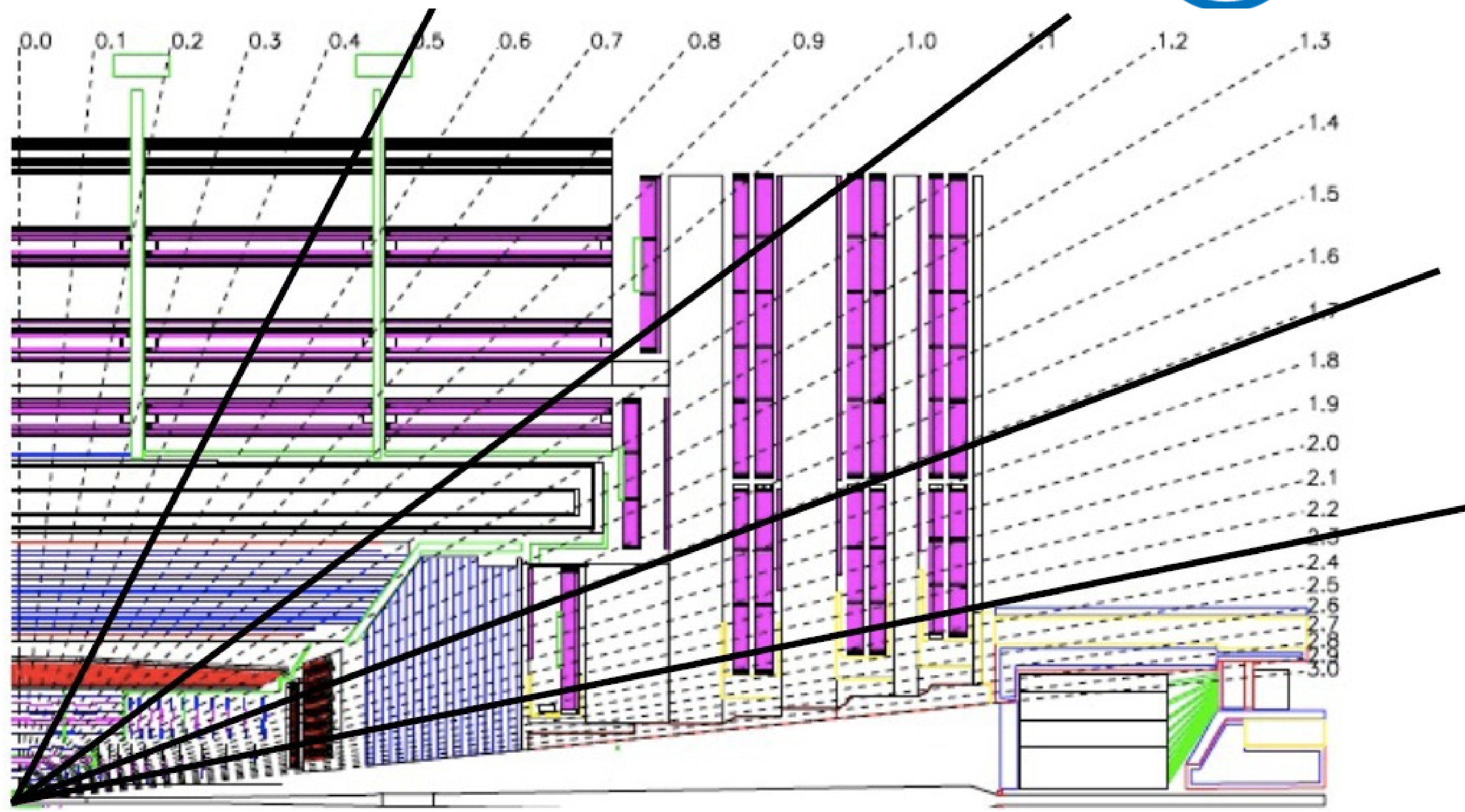

Four partitions in the CMS detector are used in the measurement of calorimeter response 


\section{Combined Calorimeter Energy Ratio (Wide Matrix)}
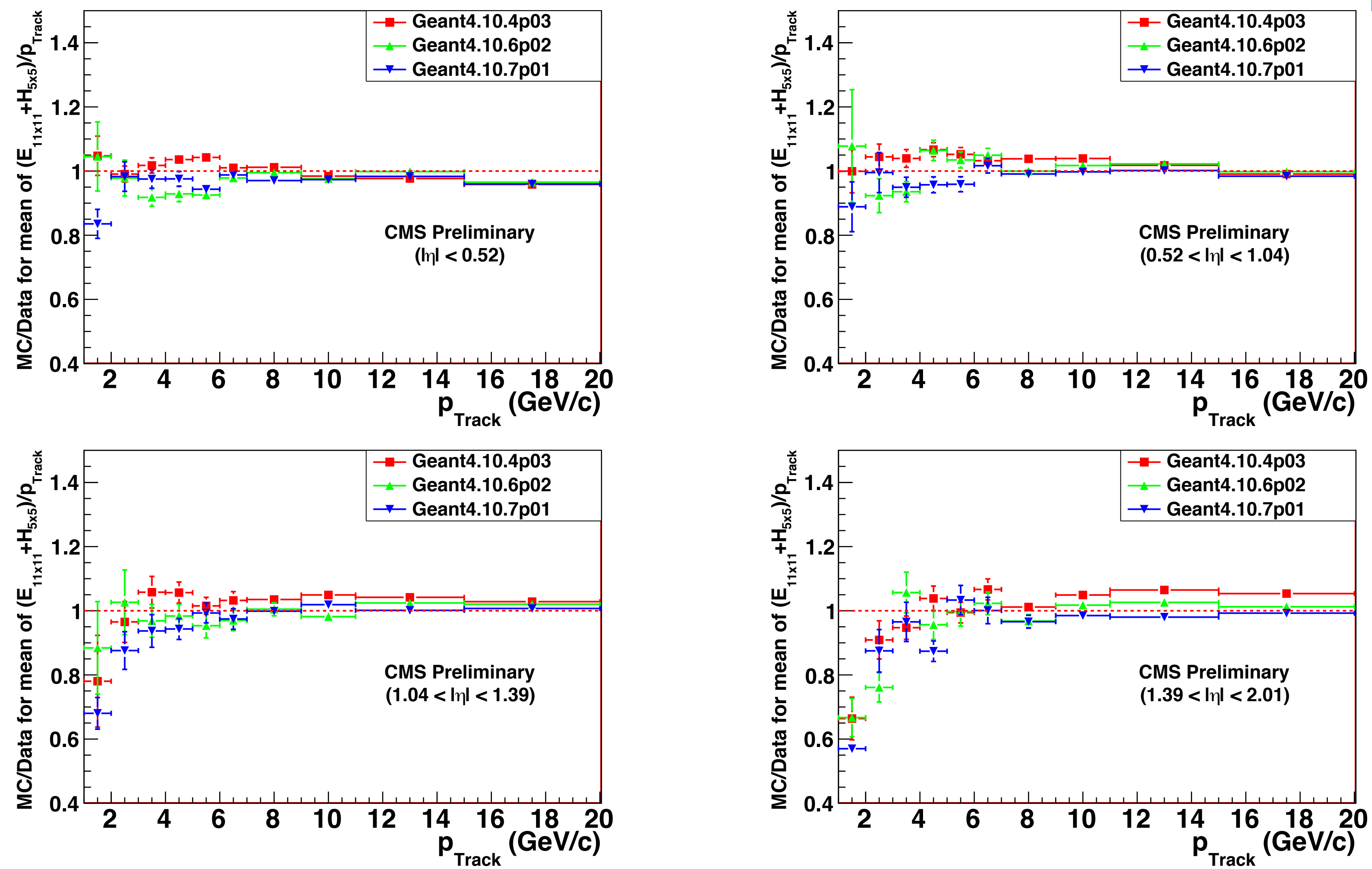

Ratio of the mean energy response in a wide matrix of ECAL and HCAL between MC and data for four regions of the calorimeter: central barrel (top left); side barrel (side barrel); transition region (bottom left); endcap (bottom right) 


\section{Level of Disagreement}

- Level of (dis)agreement is calculated from the deviation of the ratio (Data/MC) from 1.0

- The mean level of disagreement between data and $\mathrm{MC}$ is between $1.0 \%$ and $2.7 \%$ in the version 10.7.p01, depending on the region of the detector. The level of agreement is even better than in the version 10.4.p03

\begin{tabular}{|c|c|c|c|c|c|c|}
\hline & $\begin{array}{c}\left(E_{7 \times 7}+H_{3 \times 3}\right) / p \\
10.4 . p 03\end{array}$ & $\begin{array}{c}\left(E_{7 \times 7}+H_{3 \times 3}\right) / p \\
10.6 . p 02\end{array}$ & $\begin{array}{c}\left(E_{7 \times 7}+H_{3 \times 3}\right) / p \\
10.7 . p 01\end{array}$ & $\begin{array}{c}\left(E_{11 \times 11}+H_{5 \times 3}\right) / p \\
10.4 . p 03\end{array}$ & $\begin{array}{c}\left(E_{11 \times 11}+H_{5 \times 5}\right) / p \\
10.6 . p 02\end{array}$ & $\begin{array}{c}\left(E_{11 \times 11}+H_{5 \times 5}\right) / p \\
10.7 . p 01\end{array}$ \\
\hline Barrel 1 & $(1.6 \pm 0.4) \%$ & $(2.2 \pm 0.4) \%$ & $(2.7 \pm 0.4) \%$ & $(2.1 \pm 0.4) \%$ & $(2.1 \pm 0.4) \%$ & $(2.6 \pm 0.4) \%$ \\
\hline Barrel 2 & $(4.0 \pm 0.4) \%$ & $(2.5 \pm 0.4) \%$ & $(1.0 \pm 0.4) \%$ & $(2.8 \pm 0.4) \%$ & $(1.8 \pm 0.4) \%$ & $(1.0 \pm 0.4) \%$ \\
\hline Transition & $(5.3 \pm 0.5) \%$ & $(2.6 \pm 0.5) \%$ & $(1.4 \pm 0.5) \%$ & $(3.6 \pm 0.5) \%$ & $(2.1 \pm 0.5) \%$ & $(1.0 \pm 0.5) \%$ \\
\hline Endcap & $(5.5 \pm 0.5) \%$ & $(2.1 \pm 0.5) \%$ & $(2.9 \pm 0.5) \%$ & $(5.0 \pm 0.5) \%$ & $(2.3 \pm 0.5) \%$ & $(1.9 \pm 0.5) \%$ \\
\hline
\end{tabular}




\section{Summary}

- CMS has been using Geant4 as the simulation tool for comparing data with predictions from known physics models

- Geant4 has evolved over time. For most of the Run2 physics studies, the version 10.4.p03 was used. CMS has now moved to the version 10.7.p01 for Run3 physics studies

- Different Geant4 versions are tested by comparing their predictions with some controlled measurements of single particle response

- 2006 test beam data of combined CMS barrel calorimeter (prototype hadron calorimeter and electromagnetic calorimeter) and low luminosity collision data at $\sqrt{ } \mathrm{s}=13 \mathrm{TeV}$ are used for this comparison

- All 3 versions (10.4.p03, 10.6.p02, and 10.7.p01) provide good agreement with the data.

- The level of agreement is even better in the version 10.7.p01 and CMS is ready for Run3 MC production using this version 


\section{Backups}




\section{Mean response with $\pi^{+}$and anti-proton}
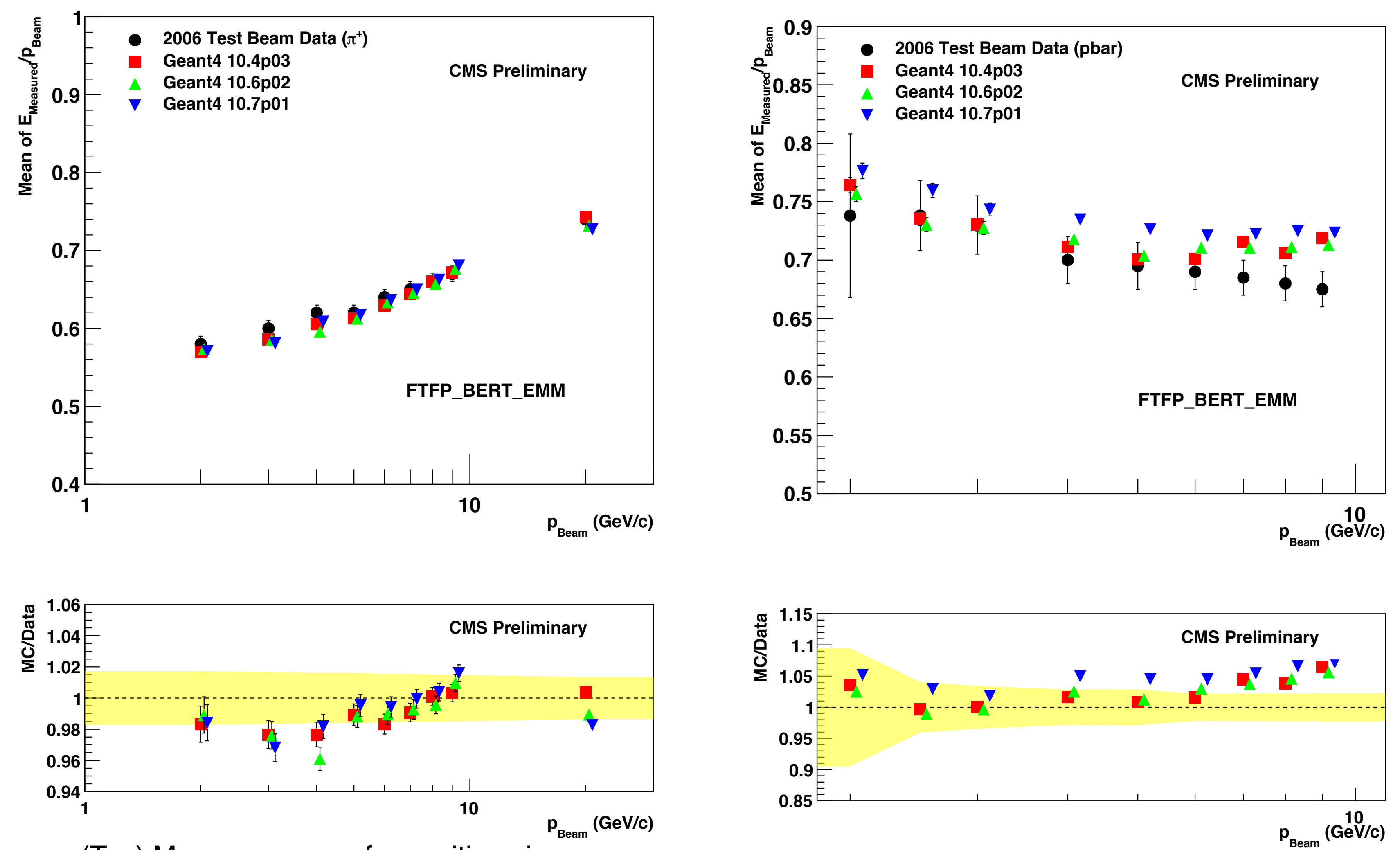

(Top) Mean response for positive pions as a function of momentum compared to $\mathrm{MC}$ predictions; (bottom) Ratio of MC to data for positive pions as a function of momentum

(Top) Mean response for anti-protons as a function of momentum compared to MC predictions; (bottom) Ratio of MC to data for antiprotons as a function of momentum 


\section{Mean Response for kaons}
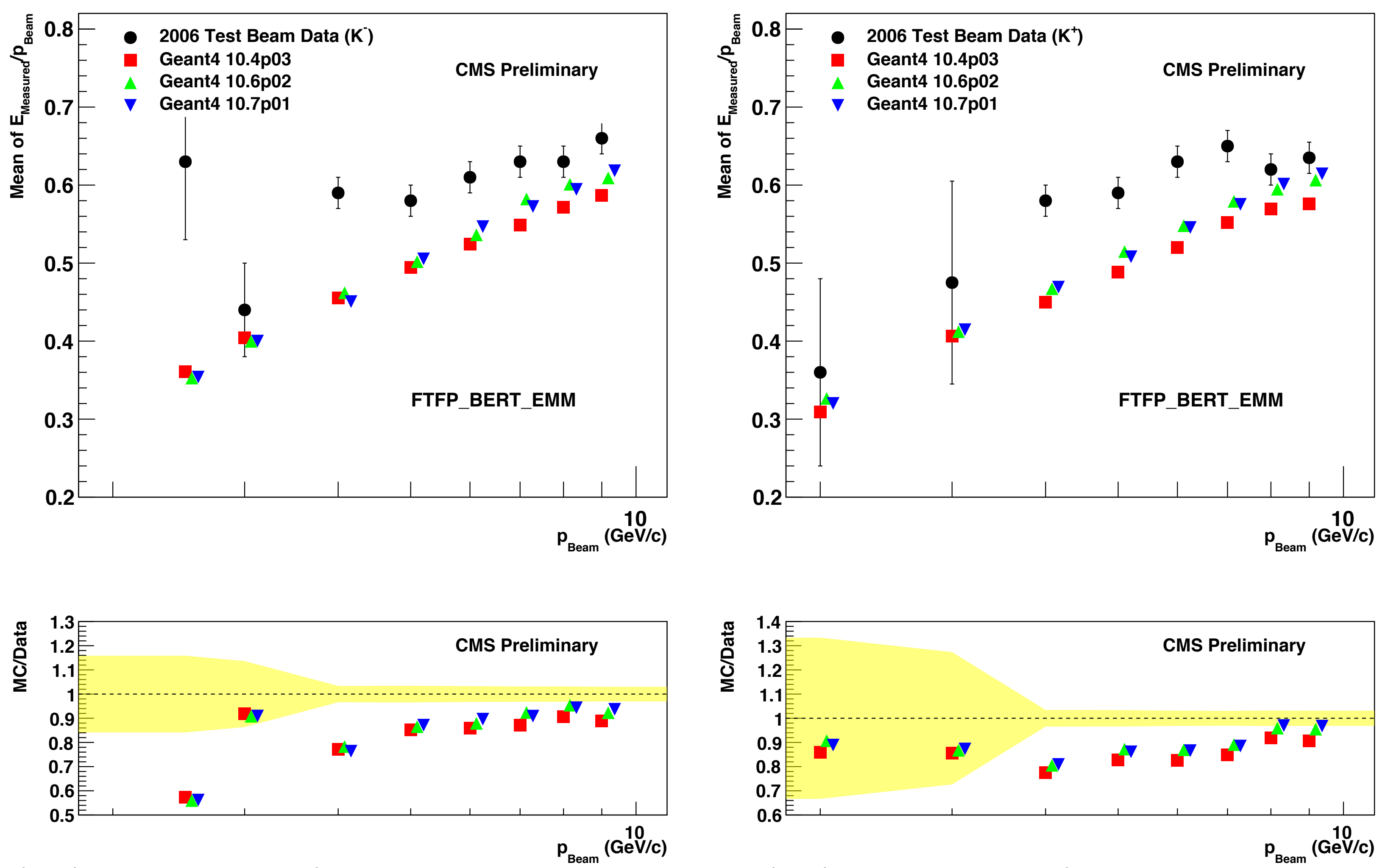

(Top) Mean response for negative kaons as a function of momentum compared to MC predictions; (bottom) Ratio of MC to data for negative kaons as a function of momentum vCHEP 2021

(Top) Mean response for positive kaons as a function of momentum compared to MC predictions; (bottom) Ratio of MC to data for positive kaons as a function of momentum

S. Banerjee, V. Ivantchenko 


\section{Energy in the ECAL ( $7 \times 7$ matrix)}
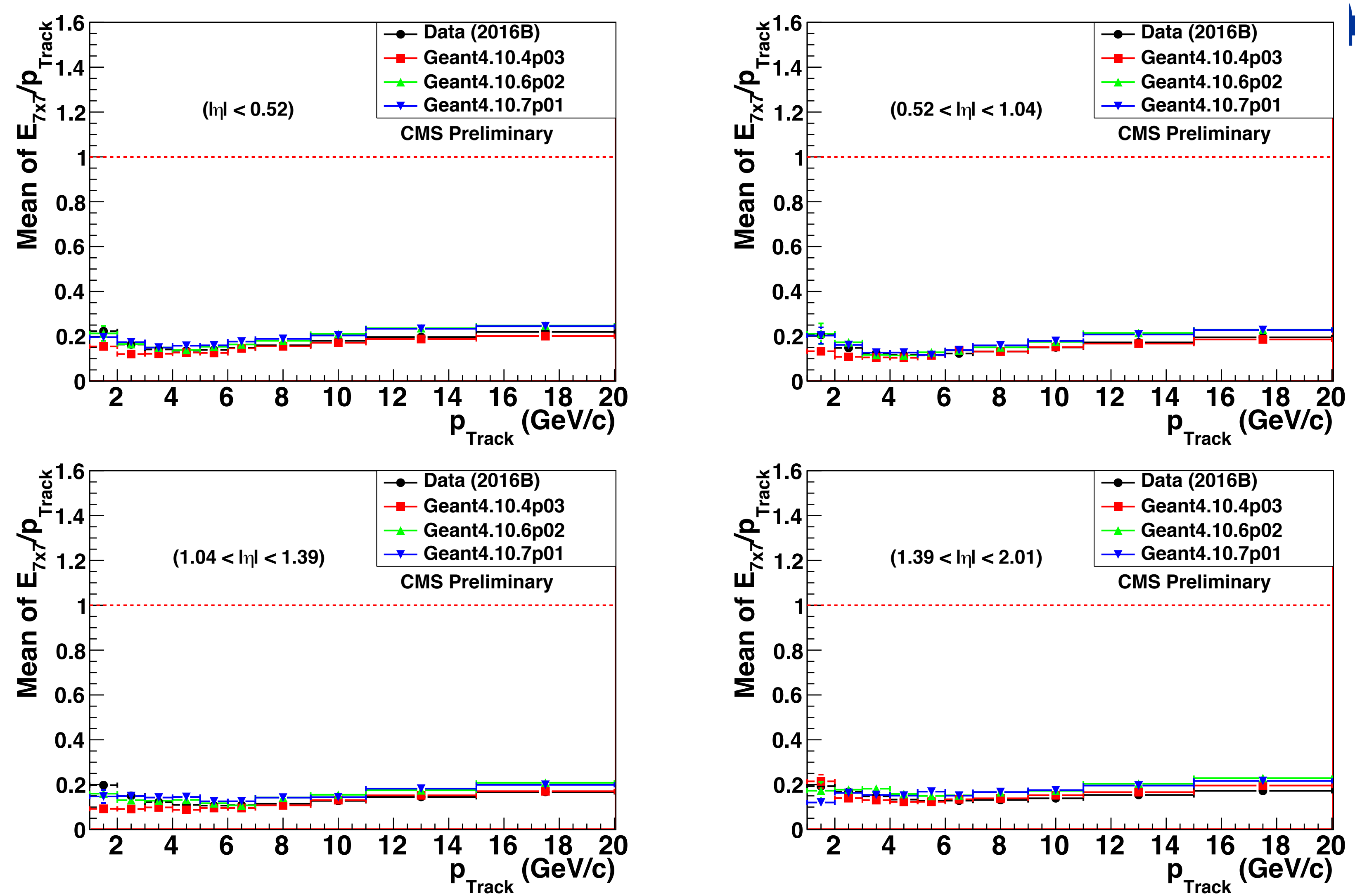

Mean of the ratio of energy measured in a $7 \times 7$ matrix in the ECAL to track momentum in 4 regions of the detector: central barrel (top left); side barrel (top right); transition region (bottom left); endcap (bottom right) 


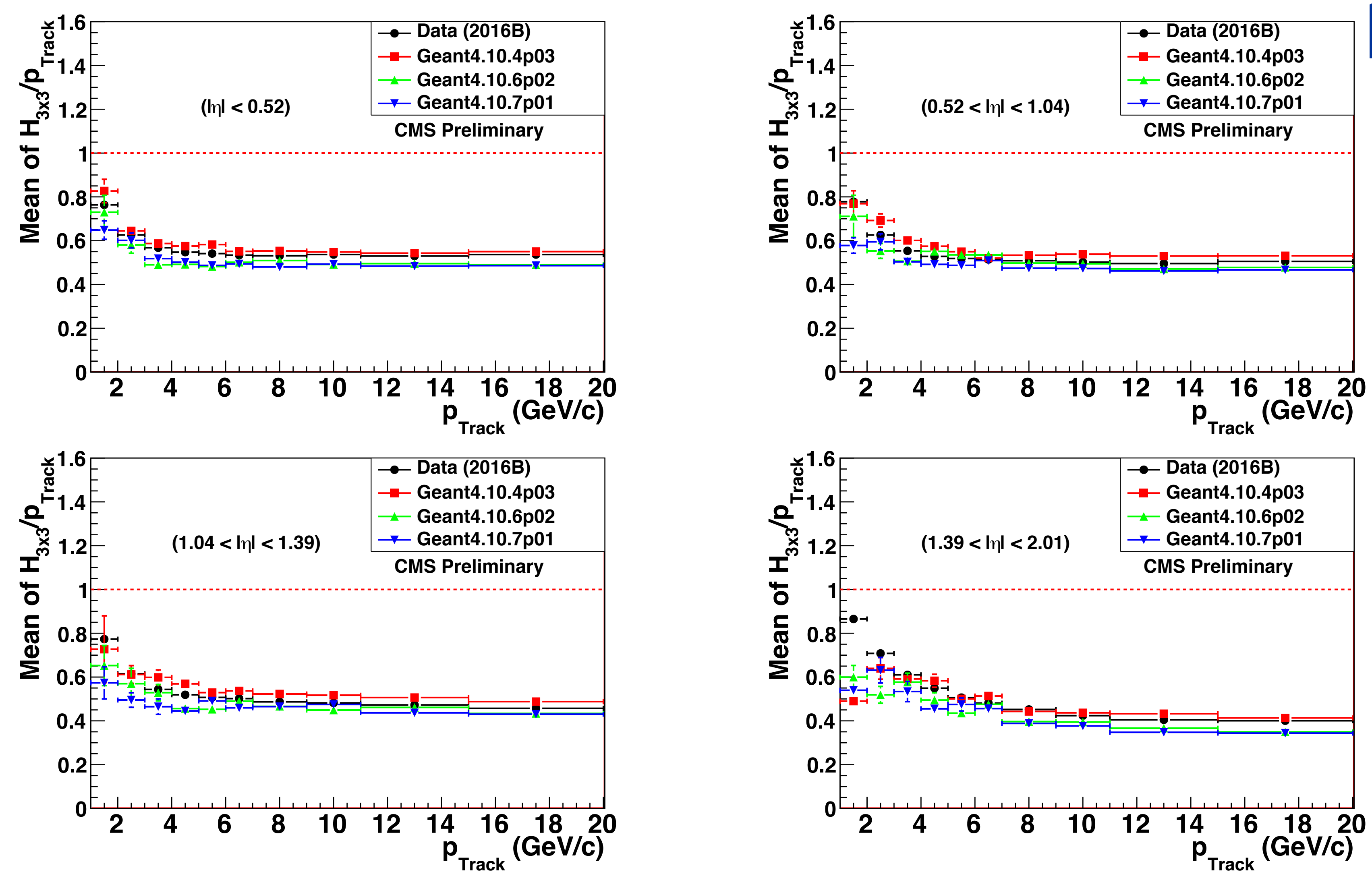

Mean of the ratio of energy measured in a 3x3 matrix in the HCAL to track momentum in 4 regions of the detector: central barrel (top left); side barrel (top right); transition region (bottom left); endcap (bottom right) 


\section{Energy in the HCAL (5x5 matrix)}
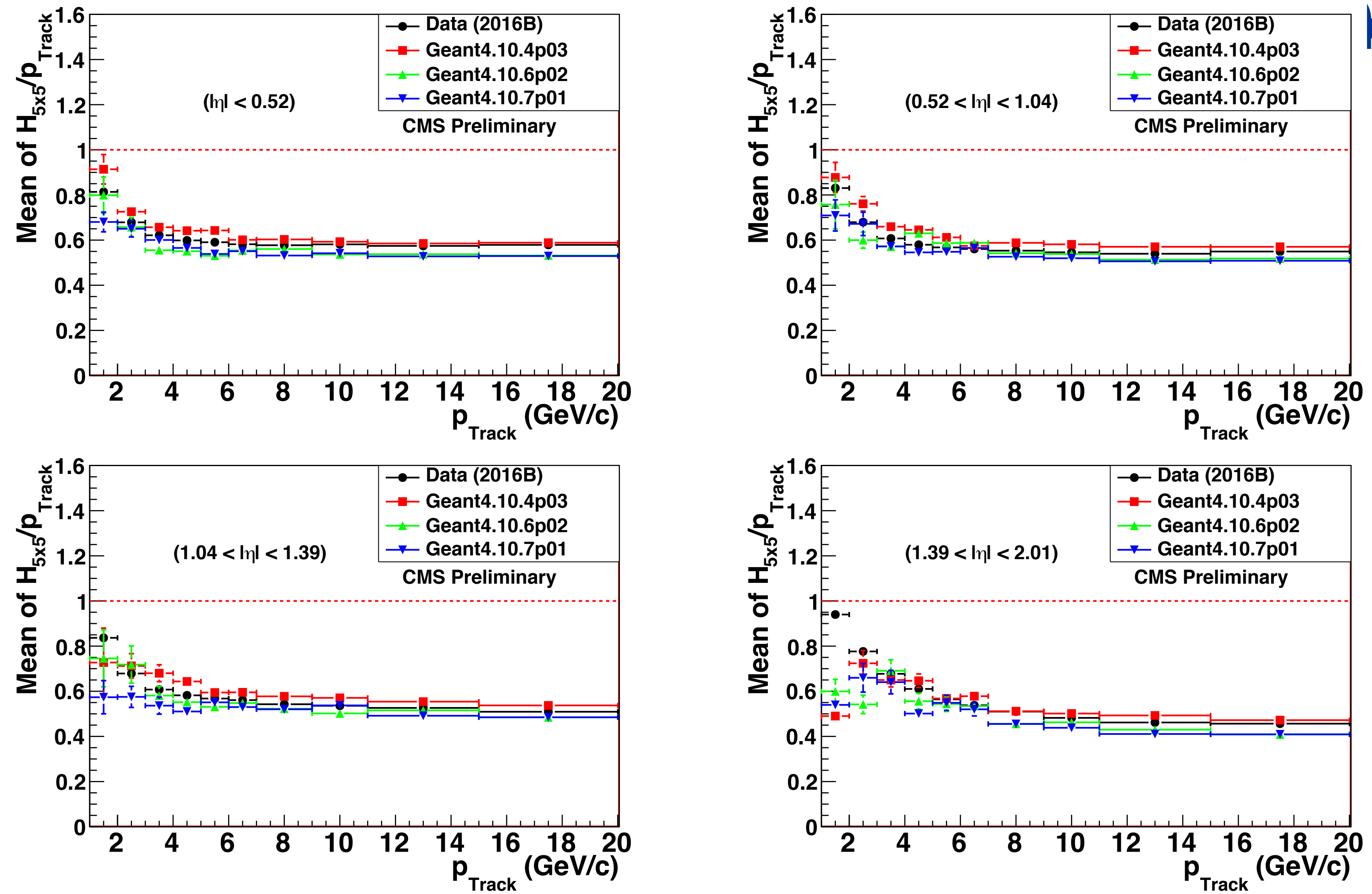

Mean of the ratio of energy measured in a $5 \times 5$ matrix of the HCAL to track momentum in 4 regions of the detector: central barrel (top left); side barrel (top right); transition region (bottom left); endcap (bottom right) 



\section{References}

[1] S. Agostinelli et. al., Nuclear Instruments and Methods A506 (2003) 250.

[2] J. Allison et. al., IEEE Transaction on Nuclear Science 53 (2006) 278.

[3] J. Allison et. al., Nuclear Instruments and Methods A835 (2016) 186.

[4] The CMS Barrel Calorimeter Response to Particle Beams from 2 to 350 GeV/c - S. Abdullin et. al., European Physical Journal C60 (2009) 359.

[5] Data/MC Comparison for ECAL and HCAL - CMS Collaboration, CMS/DP-2016/068 (2016).

[6] Validation of Physics Models of Geant4 using data from CMS Experiment

- CMS Collaboration, CMS/DP-2020/050 (2020). 